\title{
Bioanalysis
}

\section{Rapid prototyping using 3D printing in bioanalytical research}

\author{
"Advantages of 3D printing for fabrication of bioanalysis prototypes \\ include reproducibility, high precision, ease of learning, fast building \\ time, and low printing costs."
}

First draft submitted: 17 November 2016; Accepted for publication: 5 December 2016; Published online: 10 January 2017

Keywords: 3D-printed analytical devices $\bullet$ 3D printing $\bullet$ microfluidic devices $\bullet$ paper-spray cartridge $\bullet$ rapid prototyping

In bioanalytical research laboratories, 3D printing is no longer just a conception; it has become a useful tool for the fabrication of various analytical devices and custom labware in the past few years. Due to its fast designto-object workflow, ease of learning and the ability to make complex structures with sufficient resolution, 3D printing technology has shown its application in biomedical engineering, tissue scaffolding, surgical preparation, pharmacokinetics/pharmacodynamics, forensic science and medical science $[1,2]$.

Microfluidics is one of the most represented areas of 3D printing with several review articles describing the latest improvements of the fabrication of novel 3D-printed microfluidic devices. These include the integration of these devices with electrodes, biosensors and valves, and their applications in chemistry and biology [3-5], such as the analyses of cells and biomolecules as well as interfaces that enable bioanalytical measurements using cellphones [6]. Applications of 3D printing in other analytical devices have also been reported, such as 3D-printed paper spray ionization cartridge with fast wetting and continuous solvent supply features [7], 3D-printed supercapacitorpowered electrochemiluminescent for protein immunoarray [8], membrane module design with 3D printing technology [9], 3D-printed grinding device for reproducible preparation of nanospray tips [10], and 3D-printed platforms for solute delivery, separations and diagnostics [11].
There are a number of interesting examples in literature about the use of $3 \mathrm{D}$ printing in bioanalytical research, and we will highlight just a few here. 3D printing is a promising technique for developing sample-to-device interfaces for limited-resource settings and point-of-care diagnostics. Jue et al. demonstrated a 3D-printed interlock meter-mix device for metering and lysing clinical urine samples [12]. The 3D-printed static mixer contains elements designed to mix urine and lysis buffer that are injected into the device simultaneously. Rapid mixing within the first few static mixer elements was achieved. Gowers et al. described a 3D-printed microfluidic device with integrated electrode biosensors for continuous monitoring of human tissue metabolite levels, such as glucose and lactate [13]. The 3D-printed microfluidic chip and 3D-printed electrode holder in this wearable device enabled a simple connection between the microdialysis probes and electrode biosensors. In addition, a soft 3D-printed elastomer was used to ensure a good seal between electrode holder and microfluidic chip. 3D-printed devices also have been used to increase efficiency during the drug-development process. Lockwood et al. showed the parallel in vitro pharmacokinetic profiling of molecules by using a 3D-printed fluidic device [14]. The device contained multiple flow channels, and each channel was integrated with porous membranebased insert wells. The membranes enabled

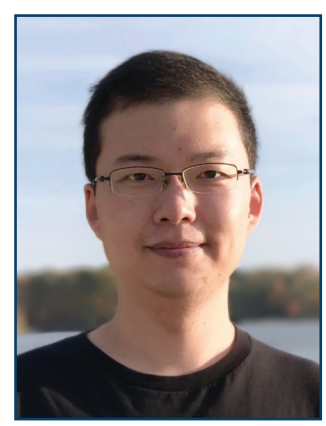

Chengsen Zhang

Author for correspondence: Department of Chemistry \& Chemical Biology, Indiana University-Purdue University Indianapolis, Indianapolis, IN 46202, USA

zhang458@iupui.edu

Brandon J Bills

Department of Chemistry \& Chemical Biology, Indiana University-Purdue University Indianapolis, Indianapolis, IN 46202, USA

Nicholas E Manicke Department of Chemistry \& Chemical Biology, Indiana University-Purdue University Indianapolis, Indianapolis, IN 46202, USA 
small-molecule drugs to diffuse back and forth between flow channels and the insert wells. Multiple pharmacokinetic profiles were generated simultaneously by using this device and the volume consumption was reduced from liters to milliliters, in comparison with diffusion-based dynamic in vitro models.

\section{"3D printing has recently attracted attention as an alternative method to fabricate analytical devices."}

In our laboratory, we are working to develop inexpensive disposable cartridges that address the entire bioanalytical workflow including sample collection, transportation/storage, sample preparation and analysis. As an example of this approach, we have been investigating paper-spray MS, in which biofluids samples are deposited and stored on paper. Extraction and ionization are then carried out directly from the dried biofluid spot on the paper without additional sample preparation (CITE). We have begun using 3D printing to generate prototype sampling cartridge as well as various devices to facilitate the experiments. This equipment is not necessarily complicated. It could be as simple as a piece of plastic to hold blood samples in a certain way while drying or more complex like a new disposable cartridge designed to perform automatic sample preconcentration.

In the past, we would manufacture these objects by using a milling machine to carve the desired piece out of blocks of plastic. Advantages of the milling machine include its relatively low cost (a quality benchtop milling machine can be purchased for $\sim$ US $\$ 1000$ ) and the wide range of materials can be machined, including metal and plastics with good solvent resistance, such as Del$\operatorname{rin}^{\circledR}$ and Teflon ${ }^{\circledR}$. However, machining parts was often time consuming, and required planning and foresight to work within the limits of what could be done with a milling machine. Parts also had to be machined one at a time. Paper spray cartridges made using a milling machine required an afternoon of tedious progress to cut out a slot for the paper using a narrow and fragile milling bit. As a result, only one or two cartridges would be made and would require cleaning between each

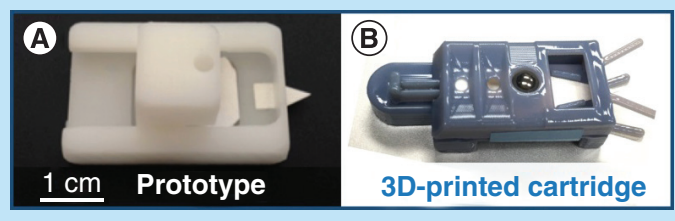

Figure 1. Paper spray cartridge integrated with solid phase extraction. (A) Prototype made by milling machine. (B) 3D-printing cartridge. sample. Recently, a service opened on campus that provided access to a number of different types and brands of $3 \mathrm{D}$ printers. Using a sufficiently high-resolution 3D printer, a cartridge with the desired dimensions can be printed in an hour. In addition, modifications to the design require only as much time as changing the $3 \mathrm{D}$ model and printing off new cartridges. This has allowed for rapid prototyping with multiple iterations and the ability to print off multiple cartridges to allow an entire experiment to be set up at once without the tedium of cleaning the cartridges between each analytical run.

In a recent experiment, for example, a special membrane had to be held against a small piece of paper while plasma wicked through from whole blood [15]. The membrane was prone to ripping so a special holder was designed capable of holding the membrane gently during the experiment. Initially the holder was machined from three pieces of plastic taking around 2 days to design and manufacture by hand. The experiment had to be modified, and the original holder no longer worked as desired. A second holder was produced using $3 \mathrm{D}$ printing. Using a free $3 \mathrm{D}$ modeling program, it took around $2 \mathrm{~h}$ to model the holder and two more hours to print five copies of the holder to scale-up the experiment. This speed and ease of making copies has proved useful in a number of experiments.

In another example, a paper spray cartridge with integrated solid phase extraction (SPE) was developed in our laboratory recently for the selective and sensitive detection of small molecule drugs in plasma [16]. The cartridge consisted of two parts that were assembled together, as shown in Figure 1A. Using a milling machine, it took us about a week to produce enough cartridges to analyze a batch of samples for quantitative analysis, in which dozens of samples needed to be prepared and tested at the same time. In addition, the milling process had to be done carefully to ensure reproducibility among cartridges. However, there is no such reproducibility issue in 3D-printed cartridges. Moreover, 3D printing speeds up the commercialization process of the SPE cartridge. In order to achieve an automatic high-throughput analysis, we redesigned the SPE cartridge to make it work in a Prosolia (IN, USA) paper spray autosampler [Unpublished Data]. The redesigned 3D-printed SPE cartridge could be printed within $2 \mathrm{~h}$, costing only US $\$ 2$. As shown in Figure 1B, the new cartridge has a smaller size in comparison with its prototype, the same position to apply spray solvent and spray voltage as a Prosolia paper spray cartridge, and is assembled from four parts with more complicated structure that would be impossible to produce by a milling machine.

Advantages of $3 \mathrm{D}$ printing for fabrication of bioanalysis prototypes include reproducibility, high preci- 
sion, ease of learning, fast building time, and low printing costs. However, there are some drawbacks to using $3 \mathrm{D}$-printed devices. Depending on the quality required, 3D printers can range in price from a few hundred dollars to tens of thousands of dollars for machines capable of fine detail. In addition, depending on the desired end-use of the 3D-printed part, solvent compatibility of the material may need to be considered. For example, the primary $3 \mathrm{D}$ printer used in our work is an Objet ${ }^{\circledR}$ printer from Stratasys ${ }^{\circledR}$ (MN, USA). This type of printer uses two types of materials, a rigid photopolymer that makes up the structure and a soluble support material to fill any gaps during the build. We have found that even with thorough cleaning, peaks in the mass spectrum originating from the support material show up during analysis using the $3 \mathrm{D}$-printed cartridges. Whether or not the material will leech, contaminants that will interfere with analysis need to be taken into consideration any time a 3D-printed sample makes direct contact with the sample.

In addition, current materials for 3D printing have shown less strength and durability, and the choice of

\section{References}

1 Gross BC, Erkal JL, Lockwood SY, Chen C, Spence DM. Evaluation of $3 \mathrm{D}$ printing and its potential impact on biotechnology and the chemical sciences. Anal. Chem. 86(7), 3240-3253 (2014).

2 Ventola CL. Medical applications for 3D printing: current and projected uses. PT 39(10), 704-711 (2014).

3 Ho CM, Ng SH, Li KHH, Yoon YJ. 3D printed microfluidics for biological applications. Lab. Chip 15(18), 3627-3637 (2015).

4 He Y, Wu Y, Fu J-Z et al. Developments of 3D printing microfluidics and applications in chemistry and biology: a review. Electroanalysis 28(8), 1658-1678 (2016).

5 Yazdi AA, Popma A, Wong W et al. 3D printing: an emerging tool for novel microfluidics and lab-on-a-chip applications. Microfluid Nanofluidics 20, 50 (2016).

6 Bishop GW, Satterwhite-Warden JE, Kadimisetty K, Rusling JF. 3D-printed bioanalytical devices. Nanotechnology 27(28), 284002 (2016).

7 Salentijn GIJ, Permentier HP, Verpoorte E. 3D-printed paper spray ionization cartridge with fast wetting and continuous solvent supply features. Anal. Chem. 86(23), 11657-11665 (2014).

8 Kadimisetty K, Mosa IM, Malla S et al. 3D-printed supercapacitor-powered electrochemiluminescent protein immunoarray. Biosens. Bioelectron. 77, 188-193 (2016).

9 Lee J-Y, Tan WS, An J et al. The potential to enhance membrane module design with 3D printing technology. J. Membr. Sci. 499, 480-490 (2016). materials available to produce functional devices is limited. Optical transparency and biocompatibility of the materials also need to be considered in some bioanalytical studies.

In conclusion, 3D printing has recently attracted attention as an alternative method to fabricate analytical devices. With the progress of $3 \mathrm{D}$ printing technology, such as more material choices, higher resolution and throughput, 3D printing has the potential to be utilized in more chemical and biological applications and change the perceived limitations in the experimental design for bioanalytical studies.

\section{Financial \& competing interests disclosure}

The authors have no relevant affiliations or financial involvement with any organization or entity with a financial interest in or financial conflict with the subject matter or materials discussed in the manuscript. This includes employment, consultancies, honoraria, stock ownership or options, expert testimony, grants or patents received or pending, or royalties.

No writing assistance was utilized in the production of this manuscript.

10 Tycova A, Prikryl J, Foret F. Reproducible preparation of nanospray tips for capillary electrophoresis coupled to mass spectrometry using $3 \mathrm{D}$ printed grinding device. Electrophoresis 37(7-8), 924-930 (2016).

11 Cabot JM, Macdonald NP, Phung SC, Breadmore MC, Paull B. Fibre-based electrofluidics on low cost versatile 3D printed platforms for solute delivery, separations and diagnostics; from small molecules to intact cells. Analyst 141(23), 6422-6431 (2016).

12 Jue E, Schoepp NG, Witters D, Ismagilov RF. Evaluating 3D printing to solve the sample-to-device interface for LRS and POC diagnostics: example of an interlock meter-mix device for metering and lysing clinical urine samples. Lab. Chip 16(10), 1852-1860 (2016).

13 Gowers SA, Curto VF, Seneci CA et al. 3D printed microfluidic device with integrated biosensors for online analysis of subcutaneous human microdialysate. Anal. Chem. 87(15), 7763-7770 (2015).

14 Lockwood SY, Meisel JE, Monsma FJ Jr, Spence DM. A diffusion-based and dynamic 3D-printed device that enables parallel in vitro pharmacokinetic profiling of molecules. Anal. Chem. 88(3), 1864-1870 (2016).

15 Bills BJ, Manicke NE. On-cartridge blood fractionation for dried plasma analysis by paper spray mass spectrometry. Clin. Mass Spectrom. 27(4), 726-734 (2016).

16 Zhang C, Manicke NE. Development of a paper spray mass spectrometry cartridge with integrated solid phase extraction for bioanalysis. Anal. Chem. 87(12), 6212-6219 (2015). 